\title{
The Bernstein Operational Matrices for Solving the Fractional Quadratic Riccati Differential Equations with the Riemann-Liouville Derivative
}

\author{
Dumitru Baleanu, ${ }^{1,2,3}$ Mohsen Alipour, ${ }^{4}$ and Hossein Jafari ${ }^{5}$ \\ ${ }^{1}$ Department of Mathematics, Cankaya University, Ogretmenler Cad. 14, Balgat, 06530 Ankara, Turkey \\ ${ }^{2}$ Institute of Space Sciences, P.O. Box MG 23, Magurele, 077125 Bucharest, Romania \\ ${ }^{3}$ Department of Chemical and Materials Engineering, Faculty of Engineering, King Abdulaziz University, P.O. Box 80204, \\ Jeddah 21589, Saudi Arabia \\ ${ }^{4}$ Faculty of Basic Science, Babol University of Technology, P.O. Box 47148-71167, Babol, Iran \\ ${ }^{5}$ Department of Mathematics, University of Mazandaran, P.O. Box 47416-95447, Babolsar, Iran
}

Correspondence should be addressed to Mohsen Alipour; m.alipour2323@gmail.com

Received 6 April 2013; Accepted 22 May 2013

Academic Editor: Ali H. Bhrawy

Copyright ( 2013 Dumitru Baleanu et al. This is an open access article distributed under the Creative Commons Attribution License, which permits unrestricted use, distribution, and reproduction in any medium, provided the original work is properly cited.

We obtain the approximate analytical solution for the fractional quadratic Riccati differential equation with the Riemann-Liouville derivative by using the Bernstein polynomials (BPs) operational matrices. In this method, we use the operational matrix for fractional integration in the Riemann-Liouville sense. Then by using this matrix and operational matrix of product, we reduce the problem to a system of algebraic equations that can be solved easily. The efficiency and accuracy of the proposed method are illustrated by several examples.

\section{Introduction}

The Riccati differential equation is named after the Italian Nobleman Count Jacopo Francesco Riccati (1676-1754). The book of Reid [1] contains the fundamental theories of the Riccati equation, with applications to random processes, optimal control, and diffusion problems. Moreover, it is well known that the one-dimensional static Schrödinger equation is closely related to a Riccati differential equation [2]. Solitary wave solution of a nonlinear partial differential equation can be represented as a polynomial in two elementary functions satisfying a projective Riccati equation [3].

In this paper, we are dealing with the fractional quadratic Riccati differential equation as follows:

$$
{ }_{a} D_{t}^{\alpha} y(t)=f(t)+b(t) y(t)+a(t) y^{2}(t), \quad a<t \leq b,
$$

subject to the initial conditions

$$
y^{(k)}(a)=y_{a}^{k}, \quad k=0,1, \ldots,\lceil\alpha\rceil-1,
$$

where $y_{a}^{k}(k=0,1, \ldots,\lceil\alpha\rceil-1)$ are constants and $a(t), b(t)$, and $f(t)$ are known functions. We can see [4] to guarantee the existence and uniqueness of the solution of (1) with initial conditions (2).

The general response expression (1) contains a parameter $\alpha$, the order of the fractional derivative that can be varied to obtain various responses. In the case that $\alpha$ is integer, then (1) is reduced to the classical Riccati differential equation.

This problem to develop the analytical and numerical method to solve the Riccati differential equation with standard derivative, the Caputo fractional derivative and the Riemann-Liouville fractional derivative, has attracted much attention and has been studied by many authors [5-18].

The aim of this work is using the Bernstein polynomials for solving the problem (1) and (2). We notice that the problem presented [18] was in the Caputo sense but in our work, the problem is with the Riemann-Liouville derivative; therefore we considered a more general space of functions. Also, in [18], the authors used the polynomials in the form of 
$B_{i, m}^{\alpha}(x)=\left(\begin{array}{c}m \\ i\end{array}\right) x^{\alpha i}\left(1-x^{\alpha}\right)^{m-i}(i=0,1, \ldots, m)$ that is different from the standard Bernstein polynomials. So, the operational matrices in this work are different from those in [18].

The organization of this paper is as follows. In Section 2, the Bernstein polynomials are introduced. Some basic definitions and properties of the fractional calculus and also the BPs operational matrix for the Riemann-Liouville fractional integration are presented in Section 3. In Section 4, by BPs operational matrices, we solve the fractional quadratic Riccati differential equation. In Section 5, we discuss the convergence of the proposed method. In Section 6, several examples are considered to evaluate the power and effectiveness of the presented method. Some conclusions are summarized in the last section.

\section{The Bernstein Polynomials and Their Properties}

On the interval $[0,1]$ we define the Bernstein polynomials (BPs) of mth degree as follows [19]:

$$
B_{i, m}(x)=\left(\begin{array}{c}
m \\
i
\end{array}\right) x^{i}(1-x)^{m-i}, \quad i=0,1, \ldots, m .
$$

Set $\left\{B_{0, m}(x), B_{1, m}(x), \ldots, B_{m, m}(x)\right\}$ in the Hilbert space $L^{2}[0,1]$ is a complete basis. We can write $\Phi_{m}(x)=A T_{m}(x)$, where $A$ is a matrix upper triangular, $T_{m}(x)=\left[1, x, \ldots, x^{m}\right]^{T}$, and $\Phi_{m}(x)=\left[B_{0, m}(x), B_{1, m}(x), \ldots, B_{m, m}(x)\right]^{T}[20]$.

As a result, any polynomial of degree $m$ can be expanded in terms of linear combination of $B_{i, m}(x)(i=0,1, \ldots, m)$ as given below:

$$
P(x)=\sum_{i=0}^{m} c_{i} B_{i, m}(x)=c^{T} \Phi_{m}(x),
$$

where

$$
c=\left(\int_{0}^{1} \Phi(x) \Phi(x)^{T} d x\right)^{-1}\left(\int_{0}^{1} P(x) \Phi(x) d x\right) .
$$

The approximation of functions within the Bernstein polynomials and convergence analysis can be found in [20, 21].

\section{BPs Operational Matrix for the Riemann-Liouville Fractional Integration}

In this section, firstly, we give some basic definitions and properties of the fractional calculus which are used further in this paper.

Definition 1 (see [4, 22-24]). Let $\alpha \geq 0$; the operator $I_{t}^{\alpha}$, defined on $L_{1}[a, b]$ by

$$
\begin{gathered}
I_{t}^{\alpha} f(t)=\frac{1}{\Gamma(\alpha)} \int_{a}^{t}(t-x)^{\alpha-1} f(x) d x, \quad a \leq t \leq b, \\
{ }_{a}^{I_{t}^{0}} f(t)=f(t),
\end{gathered}
$$

is called the Riemann-Liouville fractional integral operator of order $\alpha$.
Definition 2 (see $[4,22-24])$. Let $\alpha \geq 0$ and $n=\lceil\alpha\rceil$; the operator ${ }_{a} D_{t}^{\alpha}$, defined by

$$
\begin{gathered}
{ }_{a} D_{t}^{\alpha} f(t)=\frac{1}{\Gamma(n-\alpha)}\left(\frac{d}{d t}\right)^{n} \int_{a}^{t}(t-x)^{n-\alpha-1} f(x) d x, \\
a \leq t \leq b, \\
{ }_{a} D_{t}^{0} f(t)=f(t),
\end{gathered}
$$

is called the Riemann-Liouville fractional derivative operator of order $\alpha$.

Definition 3 (see $[4,22-24])$. Let $\alpha \geq 0, n=\lceil\alpha\rceil$, and $d^{n} f(x) / d x^{n} \in L_{1}[a, b]$. The operator ${ }_{a}^{c} D_{t}^{\alpha}$, defined by

$$
\begin{gathered}
{ }_{a}^{c} D_{t}^{\alpha} f(t)=\frac{1}{\Gamma(n-\alpha)} \int_{a}^{t}(t-x)^{n-\alpha-1} \frac{d^{n} f(x)}{d x^{n}} d x, \\
a \leq t \leq b, \\
{ }_{a}^{c} D_{t}^{0} f(t)=f(t),
\end{gathered}
$$

is called the Caputo fractional derivative operator of order $\alpha$.

Lemma 4. If $\alpha \geq 0, n=\lceil\alpha\rceil$, and $a \leq t \leq b$, then

(1) ${ }_{a}^{c} D_{t}^{\alpha} \quad I_{t}^{\alpha} f(t)=f(t)$,

(2) $a_{a} I_{t}^{\alpha} \quad{ }_{a}^{c} D_{t}^{a} f(t)=f(t)-\sum_{k=0}^{n-1} \frac{f^{(k)}(a)}{k !}(x-a)^{k}$,

(3) ${ }_{a}^{c} D_{t}^{\alpha} f(t)={ }_{a} D_{t}^{\alpha} f(t)-\sum_{k=0}^{n-1} \frac{f^{(k)}(a)}{\Gamma(k-\alpha+1)}(x-a)^{k-\alpha}$.

Proof. See [22-24].

Theorem 5. One can get BPs operational matrix $F_{\alpha}$ from order $(m+1) \times(m+1)$ for the Riemann-Liouville fractional integral as

$$
{ }_{a} I_{t}^{\alpha} \Phi_{m}(t)=\frac{1}{\Gamma(\alpha)} \int_{a}^{t}(t-x)^{\alpha-1} \Phi_{m}(x) d x \approx F_{\alpha} \Phi_{m}(t) .
$$

Proof. See [21].

\section{BPs for Solving the Fractional Quadratic Riccati Differential Equation}

Firstly, we use the initial conditions to reduce a given initialvalue problem to a problem with zero initial conditions.

So, we define

$$
y(t)=\widehat{y}(t)+z(t)
$$

where $\hat{y}(t)$ is some known function that satisfied the initial conditions (2) and $z(t)$ is a new unknown function. 
Substituting (13) in (1) and (2), we have an initial-value problem as follows:

$$
{ }_{a} D_{t}^{\alpha} z(t)=\tilde{f}(t)+\tilde{b}(t) z(t)+\widetilde{a}(t) z^{2}(t), \quad a<t \leq b,
$$

subject to the initial conditions

$$
z^{(k)}(a)=0, \quad k=0,1, \ldots,\lceil\alpha\rceil-1 .
$$

On the other hand, by (11) in Lemma 4 we can write

$$
{ }_{a}^{c} D_{t}^{\alpha} z(t)={ }_{a} D_{t}^{\alpha} z(t) .
$$

Also, by using Lemma 3.3 in [20] the inputs $\tilde{f}(t), \widetilde{a}(t), \widetilde{b}(t)$, and ${ }_{a} D_{t}^{\alpha} z(t)$ can be approximated as follows:

$$
\begin{gathered}
\tilde{f}(t) \approx F^{T} \Phi_{m}(t), \\
{ }_{a} D_{t}^{\alpha} z(t) \approx C^{T} \Phi_{m}(t), \\
\tilde{a}(t) \approx A^{T} \Phi_{m}(t), \\
\tilde{b}(t) \approx B^{T} \Phi_{m}(t),
\end{gathered}
$$

where $F, A$, and $B$ are known $(m+1)$ column vectors and $C$ is an unknown $(m+1)$ column vector.

From (10), (15), (16), (18), and (12), we have $z(t)$

$$
\begin{aligned}
& \underset{(10)}{=} a^{\alpha} I_{t}^{\alpha}{ }_{a}^{c} D_{t}^{\alpha} z(t)+\sum_{k=0}^{n-1} \frac{z^{(k)}(a)}{k !}(x-a)^{k} \underset{(15)}{=} a_{a} I_{t}^{\alpha}{ }_{a}^{c} D_{t}^{\alpha} z(t) \\
& \underset{(16)}{=} I^{\alpha}{ }_{a}^{\alpha} D_{t}^{\alpha} z(t) \underset{(18)}{\approx} I_{t}^{\alpha}\left(C^{T} \Phi_{m}(t)\right) \\
& =C^{T}{ }_{a} I_{t}^{\alpha} \Phi_{m}(t) \underset{(12)}{\approx} C^{T} F_{\alpha} \Phi_{m}(t) \\
& =C_{\alpha}^{T} \Phi_{m}(t),
\end{aligned}
$$

where $C_{\alpha}^{T}=C^{T} F_{\alpha}$.

Now, by substituting (17)-(21) into (14), we obtain

$$
\begin{aligned}
C^{T} \Phi_{m}(t)= & F^{T} \Phi_{m}(t)+B^{T} \Phi_{m}(t) \Phi_{m}(t)^{T} F_{\alpha}^{T} C+A^{T} \Phi_{m}(t) \\
& \times\left(C^{T} F_{\alpha} \Phi_{m}(t) \Phi_{m}(t)^{T} F_{\alpha}^{T} C\right) \\
= & F^{T} \Phi_{m}(t)+B^{T} \Phi_{m}(t) \Phi_{m}(t)^{T} C_{\alpha}+A^{T} \Phi_{m}(t) \\
& \times\left(C_{\alpha}^{T} \Phi_{m}(t) \Phi_{m}(t)^{T} C_{\alpha}\right)
\end{aligned}
$$

Then, from Lemma 3.5 in [20] we have

$$
\begin{aligned}
& C_{\alpha}^{T} \Phi_{m}(x) \Phi_{m}(x)^{T} \approx \Phi_{m}(x)^{T} \widetilde{C}_{\alpha} \\
& A^{T} \Phi_{m}(x) \Phi_{m}(x)^{T} \approx \Phi_{m}(x)^{T} \widehat{A} \\
& B^{T} \Phi_{m}(x) \Phi_{m}(x)^{T} \approx \Phi_{m}(x)^{T} \widehat{B} .
\end{aligned}
$$

Therefore we can reduce (22) by (23)-(25) as

$$
C^{T} \Phi_{m}(t)=F^{T} \Phi_{m}(t)+\Phi_{m}(t)^{T} \widehat{B} C_{\alpha}+\Phi_{m}(t)^{T} \widehat{A} \widetilde{C}_{\alpha} C_{\alpha}
$$

Finally, we obtain the following nonlinear system of algebraic equation:

$$
C=F+\widehat{B} C_{\alpha}+\widehat{A} \widetilde{C}_{\alpha} C_{\alpha}
$$

such that by solving this system we can obtain the vector $C$. Then, we can get

$$
y(t) \approx \widehat{y}(t)+C^{T} F_{\alpha} \Phi_{m}(t)
$$

\section{Convergence Analysis}

In this section, we investigate the convergence analysis for the method presented in Section 4.

The problem (14) changes to the following problems

$$
\begin{aligned}
{ }_{a} D_{t}^{\alpha} z(t)= & \tilde{f}(t)+\widetilde{b}(t)\left({ }_{a} I_{t}^{\alpha}{ }_{a} D_{t}^{\alpha} z(t)\right) \\
& +\widetilde{a}(t)\left({ }_{a} I_{t}^{\alpha}{ }_{a} D_{t}^{\alpha} z(t)\right)^{2}, \quad a<t \leq b,
\end{aligned}
$$

since

$$
\begin{array}{r}
z(t) \underset{(10)}{=} a^{\prime} I_{t}^{\alpha} \quad{ }_{a}^{c} D_{t}^{\alpha} z(t)+\sum_{k=0}^{n-1} \frac{z^{(k)}(a)}{k !}(x-a)^{k} \\
\underset{(15)}{=} a^{I_{t}^{\alpha}} \quad{ }_{a}^{c} D_{t}^{\alpha} z(t) \underset{(16)}{=} a_{t} I_{a}^{\alpha} D_{t}^{\alpha} z(t) .
\end{array}
$$

By taking $u(t)={ }_{a} D_{t}^{\alpha} z(t)$ we obtain the following fractional integral equation:

$$
\begin{array}{r}
u(t)=\tilde{f}(t)+\widetilde{b}(t){ }_{a} I_{t}^{\alpha} u(t)+\widetilde{a}(t)\left({ }_{a} I_{t}^{\alpha} u(t)\right)^{2}, \\
a<t \leq b .
\end{array}
$$

If we use the approximation $u(t) \approx C^{T} \Phi_{m}(t)$, then the problem (31) from space $C^{1}[0,1]$ reduces to the following problem in space $S_{m}=\operatorname{Span}\left\{B_{0, m}(t), B_{1, m}(t), \ldots, B_{m, m}(t)\right\}$ :

$$
\begin{aligned}
C^{T} \Phi_{m}(t)= & \tilde{f}(t)+\widetilde{b}(t){ }_{a} I_{t}^{\alpha}\left(C^{T} \Phi_{m}(t)\right) \\
& +\widetilde{a}(t)\left({ }_{a} I_{t}^{\alpha}\left(C^{T} \Phi_{m}(t)\right)\right)^{2}, \quad a<t \leq b .
\end{aligned}
$$

Now, similar to Theorem 6.1 in [21], we propose the next theorem.

Theorem 6. Suppose that $u^{*}(t) \in C^{1}[0,1]$ is the exact solution of (31) and $\mu_{m}=J\left[u_{m}\right]=\operatorname{Min}_{u \in S_{m}} J[u]$, where

$$
J[u]=\left\|u(t)-\tilde{f}(t)-\tilde{b}(t){ }_{a} I_{t}^{\alpha} u(t)-\tilde{a}(t)\left({ }_{a} I_{t}^{\alpha} u(t)\right)^{2}\right\| .
$$

Then one has $\mu_{m} \rightarrow 0$ as $m \rightarrow \infty$ (i.e., $u_{m}(t) \rightarrow u^{*}(t)$ as $m \rightarrow \infty)$. 
Proof. By substituting (33) instead of (41) in [21], we can use the proof of Theorem 6.1 in [21].

Theorem 7. Suppose that $u^{*}(t) \in C^{1}[0,1]$ is the exact solution of (31) and $u_{m}(t) \in S_{m}$ is the obtained solution of (26). Then one has $u_{m}(t) \rightarrow u^{*}(t)$ as $m \rightarrow \infty$.

Proof. Substituting (17), (19), (20), and (12) in (32) we have

$$
\begin{aligned}
C^{T} \Phi_{m}(t)= & \left(F^{T} \Phi_{M}(t)+e_{\tilde{f}}^{M}\right) \\
& +\left(B^{T} \Phi_{M}(t)+e_{\tilde{b}}^{M}\right)\left(C^{T}\left(F_{\alpha} \Phi_{M}(t)+E_{I}^{M}\right)\right) \\
& +\left(A^{T} \Phi_{M}(t)+e_{\tilde{a}}^{M}\right)\left(C^{T}\left(F_{\alpha} \Phi_{M}(t)+E_{I}^{M}\right)\right)^{2}
\end{aligned}
$$

From Lemmas 2.3 and 4.1 in [21], we have $e_{\widetilde{f}}^{M}, e_{\widetilde{b}}^{M}, e_{\widetilde{a}}^{M}, E_{I}^{M} \rightarrow$ 0 as $M \rightarrow \infty$. So we can observe that as $M$ increases, (34) gets close to (32). Now, by taking $M=m$ we propose the following problem that gets close to (32) as $m$ increases:

$$
\begin{aligned}
C^{T} \Phi_{m}(t)= & F^{T} \Phi_{m}(t)+B^{T} \Phi_{m}(t) \Phi_{m}(t)^{T} C_{\alpha} \\
& +A^{T} \Phi_{m}(t)\left(C_{\alpha}^{T} \Phi_{m}(t) \Phi_{m}(t)^{T} C_{\alpha}\right)
\end{aligned}
$$

Then by (23) and (25), (35) reduces to the following equation:

$$
\begin{aligned}
C^{T} \Phi_{m}(t)= & F^{T} \Phi_{m}(t)+\left(\Phi_{M}(t)^{T} \widehat{B}+\widehat{E}_{B}^{M}\right) C_{\alpha} \\
& +A^{T} \Phi_{m}(t)\left(\left(\Phi_{M}(t)^{T} \widetilde{C}_{\alpha}+\widetilde{E}_{C_{\alpha}}^{M}\right) C_{\alpha}\right) .
\end{aligned}
$$

Equation (36) gets to (35) as $M \rightarrow \infty$, because from Lemma 3.1 in [21] $\widehat{E}_{B}^{M}, \widetilde{E}_{C_{\alpha}}^{M} \rightarrow 0$ as $M \rightarrow \infty$. Then by deleting $\widehat{E}_{B}^{M}, \widetilde{E}_{C_{\alpha}}^{M}$, taking $M=m$, and using (24) in (36), we have

$$
\begin{aligned}
C^{T} \Phi_{m}(t)= & F^{T} \Phi_{m}(t)+\Phi_{m}(t)^{T} \widehat{B} C_{\alpha} \\
& +\left(\Phi_{M}(t)^{T} \widehat{A}+\widehat{E}_{A}^{M}\right) \widetilde{C}_{\alpha} C_{\alpha}
\end{aligned}
$$

where, from Lemma 3.1 in [21], $\widehat{E}_{A}^{M} \rightarrow 0$ as $M \rightarrow \infty$. Now, by taking $M=m$ and deleting $\widehat{E}_{A}^{M}$ in (37), we get (26). Obviously, if $\tilde{u}_{m}(t)$ is solution of (32), then we have $\tilde{u}_{m}-u_{m} \rightarrow 0$ as $m \rightarrow \infty$.

On the other hand, from Theorem 6 we obtained $\tilde{u}_{m} \rightarrow$ $u^{*}$ as $m \rightarrow \infty$. Therefore we can write $u_{m} \rightarrow u^{*}$ as $m \rightarrow \infty$ and the proof is complete.

\section{Illustrative Numerical Examples}

In this section, we apply our method with $m=10$ (BPs of degree $m=10)$ to solve the following examples. We define $y_{m}(t)$ and $y(t)$ for the approximate solution and the exact solution, respectively.

Example 1. Consider the nonlinear Riccati differential equation [14]:

$$
{ }_{0} D_{t}^{\alpha} y(t)=-y(t)^{2}+1, \quad 0<t \leq 1
$$

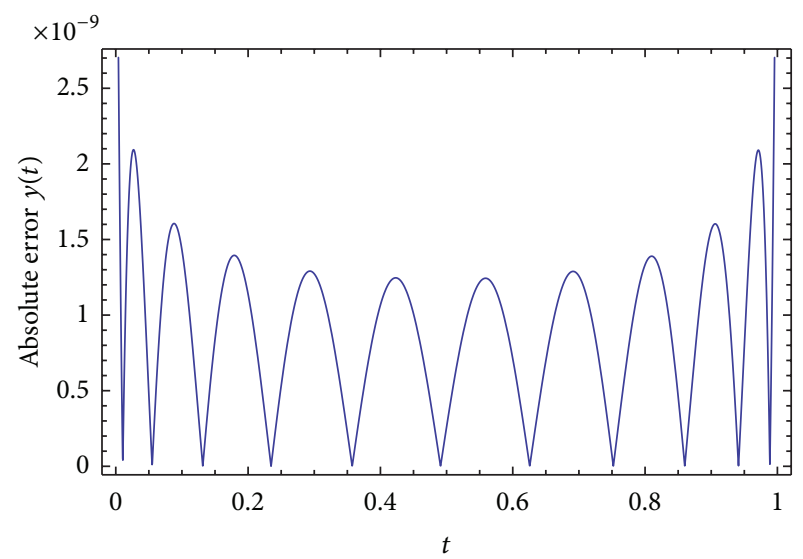

FIGURE 1: Behavior of the absolute error function in Example 1 for $\alpha=1$ and $m=10$.

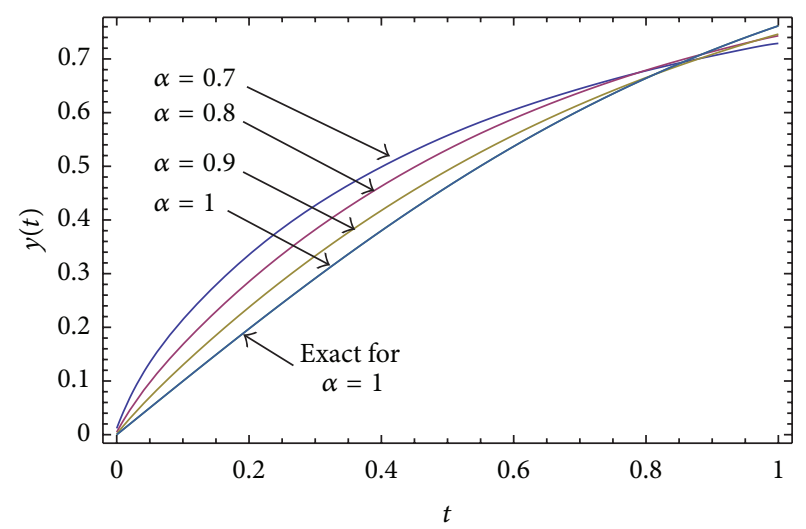

Figure 2: Behavior of $y_{10}(t)$ in Example 1 for different $\alpha$ and exact solution for $\alpha=1$.

subject to the initial condition as $y(0)=0$. The exact solution of the equation for $\alpha=1$ is given as

$$
y(t)=\frac{e^{2 t}-1}{e^{2 t}+1}
$$

Numerical results compared to [14] are given in Table 1 and also Figure 1 shows the absolute error for our method for $\alpha=$ 1 and Figure 2 shows behavior $y_{10}(t)$ for different values of $\alpha$.

Example 2. Consider the following quadratic Riccati differential equation of fractional order [14]

$$
{ }_{0} D_{t}^{\alpha} y(t)=2 y(t)-y(t)^{2}+1, \quad 0<t \leq 1,
$$

subject to the initial condition as $y(0)=0$. The exact solution of the equation for $\alpha=1$ is given as

$$
y(t)=1+\sqrt{2} \tanh \left(\sqrt{2} t+\frac{1}{2} \log \left(\frac{\sqrt{2}-1}{\sqrt{2}+1}\right)\right) .
$$

Numerical results compared to [14] are given in Table 2 and also, Figure 3 shows the absolute error for our method for $\alpha=$ 1 and Figure 4 shows behavior $y_{10}(t)$ for different values of $\alpha$. 
TABLE 1: Numerical results for $\alpha=1$ and $m=10$ in Example 1 with comparison to exact solution and [14].

\begin{tabular}{lccc}
\hline$t$ & Exact & $\begin{array}{c}\text { Present method } \\
m=10\end{array}$ & Reference [14] \\
\hline 0.1 & 0.099668 & 0.099668 & 0.099668 \\
0.2 & 0.197375 & 0.197375 & 0.197375 \\
0.3 & 0.291313 & 0.291313 & 0.291313 \\
0.4 & 0.379949 & 0.379949 & 0.379944 \\
0.5 & 0.462117 & 0.462117 & 0.462078 \\
0.6 & 0.537050 & 0.537050 & 0.536857 \\
0.7 & 0.604368 & 0.604368 & 0.603631 \\
0.8 & 0.664037 & 0.664037 & 0.661706 \\
0.9 & 0.716298 & 0.716298 & 0.709919 \\
1 & 0.761594 & 0.761594 & 0.746032 \\
\hline
\end{tabular}

TABLE 2: Numerical results for $\alpha=1$ and $m=10$ in Example 2 with comparison to exact solution and [14].

\begin{tabular}{lccc}
\hline$t$ & Exact & $\begin{array}{c}\text { Present method } \\
m=10\end{array}$ & Reference [14] \\
\hline 0.1 & 0.110295 & 0.110295 & 0.110294 \\
0.2 & 0.241977 & 0.241977 & 0.241965 \\
0.3 & 0.395105 & 0.395105 & 0.395106 \\
0.4 & 0.567812 & 0.567812 & 0.568115 \\
0.5 & 0.756014 & 0.756014 & 0.757564 \\
0.6 & 0.953566 & 0.953566 & 0.958259 \\
0.7 & 1.152949 & 1.152949 & 1.163459 \\
0.8 & 1.346364 & 1.346364 & 1.365240 \\
0.9 & 1.526911 & 1.526911 & 1.554960 \\
1 & 1.689499 & 1.689499 & 1.723810 \\
\hline
\end{tabular}

TABLE 3: Numerical results for $\alpha=1.5$ in Example 3 with comparison to [25-27].

\begin{tabular}{lcccc}
\hline$t$ & $\begin{array}{c}\text { Present method } \\
m=10\end{array}$ & ADM [25] & FDTM [26] & BPFs [27] \\
\hline 0.1 & 0.023779 & 0.023790 & 0.023790 & 0.023800 \\
0.2 & 0.067336 & 0.067330 & 0.067330 & 0.067335 \\
0.3 & 0.123886 & 0.123896 & 0.123896 & 0.123900 \\
0.4 & 0.191373 & 0.191362 & 0.191362 & 0.191368 \\
0.5 & 0.268851 & 0.268856 & 0.268856 & 0.268862 \\
0.6 & 0.356235 & 0.356238 & 0.356238 & 0.356244 \\
0.7 & 0.453958 & 0.453950 & 0.453950 & 0.453956 \\
0.8 & 0.562999 & 0.563007 & 0.563007 & 0.563014 \\
0.9 & 0.685066 & 0.685056 & 0.685056 & 0.685067 \\
1 & 0.822540 & 0.822511 & 0.822509 & 0.822525 \\
\hline
\end{tabular}

Example 3. Consider the nonlinear fractional differential equation:

$$
{ }_{0} D_{t}^{\alpha} y(t)=y^{2}(t)+1, \quad 0<t \leq 1, n-1<\alpha \leq n,
$$

TABLE 4: Numerical results for $\alpha=2.5$ in Example 3 with comparison to $[25,26]$.

\begin{tabular}{lccc}
\hline$t$ & $\begin{array}{c}\text { Present method } \\
m=10\end{array}$ & ADM [25] & FDTM [26] \\
\hline 0.1 & 0.000952 & 0.000952 & 0.000952 \\
0.2 & 0.005383 & 0.005383 & 0.005383 \\
0.3 & 0.014833 & 0.014833 & 0.014833 \\
0.4 & 0.030450 & 0.030450 & 0.030450 \\
0.5 & 0.053197 & 0.053197 & 0.053197 \\
0.6 & 0.083925 & 0.083925 & 0.083925 \\
0.7 & 0.123412 & 0.123412 & 0.123412 \\
0.8 & 0.172391 & 0.172391 & 0.172391 \\
0.9 & 0.231574 & 0.231574 & 0.231574 \\
1 & 0.301676 & 0.301676 & 0.301676 \\
\hline
\end{tabular}

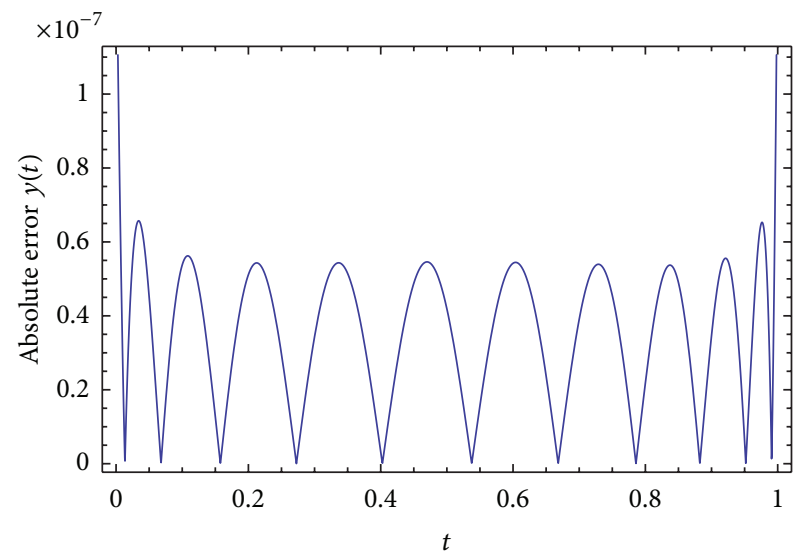

FIgURE 3: Behavior of the absolute error function in Example 2 for $\alpha=1$ and $m=10$.

with the initial conditions

$$
y^{(k)}(0)=0, \quad k=0,1, \ldots, n-1 .
$$

This problem has been studied by using ADM [25], FDTM [26], and BPFs [27]. Our results with $\alpha=1.5, \alpha=2.5$ are compared to [25-27] in Tables 3 and 4. Therefore, we see that our method is very effective and obtained solutions that are in good agreement with the results in [25-27]. Also, Figure 5 shows behavior $y_{m}(t)$ for different values of $\alpha$.

\section{Conclusion}

In this paper, we proposed a numerical method for solving the fractional quadratic Riccati differential equations by the operational matrices of the Bernstein polynomials. We applied operational matrix for fractional integration in the Riemann-Liouville sense. Then by using this matrix and operational matrix of product, we reduced the fractional quadratic Riccati differential equation to a system of algebraic equations that can be solved easily. Finally, examples have been simulated to demonstrate the high performance of the proposed method. We saw that the results were in good 


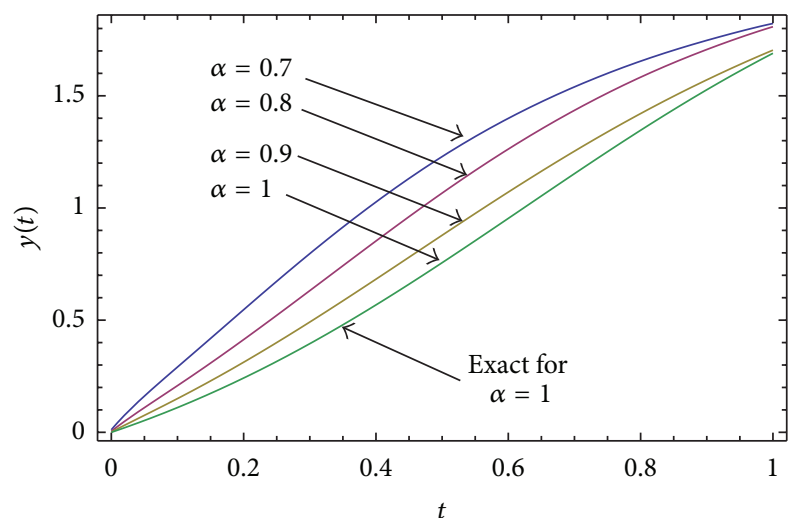

Figure 4: Behavior of $y_{10}(t)$ in Example 2 for different $\alpha$ and exact solution for $\alpha=1$.

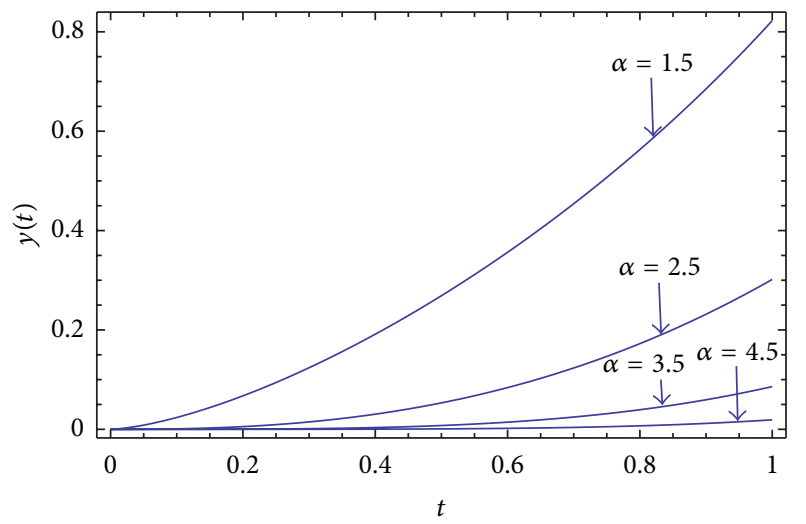

Figure 5: Plot of $y_{10}(t)$ for different $\alpha$ in Example 3.

agreement with the analytical solutions and the solutions in the open literatures. Also, we observed that the solutions approach to classical solutions as the order of the fractional derivatives approaches 1 , for fixed $m$.

\section{References}

[1] W. T. Reid, Riccati Differential Equations, vol. 86, Academic Press, New York, NY, USA, 1972, Mathematics in Science and Engineering.

[2] V. V. Kravchenko, Applied Pseudoanalytic Function Theory, Frontiers in Mathematics, Birkhäuser, Basel, Switzerland, 2009.

[3] R. Conte and M. Musette, "Link between solitary waves and projective Riccati equations," Journal of Physics A, vol. 25, no. 21, pp. 5609-5623, 1992.

[4] K. Diethelm, The Analysis of Fractional Differential Equations: An Application-Oriented Exposition Using Differential Operators of Caputo Type, vol. 2004 of Lecture Notes in Mathematics, Springer, Berlin, Germany, 2010.

[5] S. Abbasbandy, "Iterated He's homotopy perturbation method for quadratic Riccati differential equation," Applied Mathematics and Computation, vol. 175, no. 1, pp. 581-589, 2006.

[6] S. Abbasbandy, "Homotopy perturbation method for quadratic Riccati differential equation and comparison with Adomian's decomposition method," Applied Mathematics and Computation, vol. 172, no. 1, pp. 485-490, 2006.
[7] S. Abbasbandy, "A new application of He's variational iteration method for quadratic Riccati differential equation by using Adomian's polynomials," Journal of Computational and Applied Mathematics, vol. 207, no. 1, pp. 59-63, 2007.

[8] Y. Tan and S. Abbasbandy, "Homotopy analysis method for quadratic Riccati differential equation," Communications in Nonlinear Science and Numerical Simulation, vol. 13, no. 3, pp. 539-546, 2008.

[9] F. Geng, Y. Lin, and M. Cui, "A piecewise variational iteration method for Riccati differential equations," Computers \& Mathematics with Applications, vol. 58, no. 11-12, pp. 2518-2522, 2009.

[10] F. Z. Geng and X. M. Li, "A new method for Riccati differential equations based on reproducing kernel and quasilinearization methods," Abstract and Applied Analysis, vol. 2012, Article ID 603748, 8 pages, 2012.

[11] N. A. Khan, A. Ara, and M. Jamil, "An efficient approach for solving the Riccati equation with fractional orders," Computers \& Mathematics with Applications, vol. 61, no. 9, pp. 2683-2689, 2011.

[12] S. Momani and N. Shawagfeh, "Decomposition method for solving fractional Riccati differential equations," Applied Mathematics and Computation, vol. 182, no. 2, pp. 1083-1092, 2006.

[13] J. Cang, Y. Tan, H. Xu, and S.-J. Liao, "Series solutions of non-linear Riccati differential equations with fractional order," Chaos, Solitons and Fractals, vol. 40, no. 1, pp. 1-9, 2009.

[14] Z. Odibat and S. Momani, "Modified homotopy perturbation method: application to quadratic Riccati differential equation of fractional order," Chaos, Solitons and Fractals, vol. 36, no. 1, pp. 167-174, 2008.

[15] C. Li and Y. Wang, "Numerical algorithm based on Adomian decomposition for fractional differential equations," Computers \& Mathematics with Applications, vol. 57, no. 10, pp. 1672-1681, 2009.

[16] H. Jafari and H. Tajadodi, "He's variational iteration method for solving fractional Riccati differential equation," International Journal of Differential Equations, vol. 2010, Article ID 764738, 8 pages, 2010.

[17] M. Merdan, "On the solutions fractional Riccati differential equation with modified Riemann-Liouville derivative," International Journal of Differential Equations, vol. 2012, Article ID 346089, 17 pages, 2012.

[18] S. Yüzbaşı, "Numerical solutions of fractional Riccati type differential equations by means of the Bernstein polynomials," Applied Mathematics and Computation, vol. 219, no. 11, pp. 6328-6343, 2013.

[19] E. W. Cheney, Introduction to Approximation Theory, AMS Chelsea, Providence, RI, USA, 2nd edition, 1998.

[20] M. Alipour, D. Rostamy, and D. Baleanu, "Solving multidimensional FOCPs with inequality constraint by BPs operational matrices," Journal of Vibration and Control, 2012.

[21] D. Rostamy, M. Alipour, H. Jafari, and D. Baleanu, "Solving multi-term orders fractional differential equations by operational matrices of BPs with convergence analysis," Romanian Reports in Physics, vol. 65, no. 2, pp. 334-349, 2013.

[22] I. Podlubny, Fractional Differential Equations, vol. 198, Academic Press, New York, NY, USA, 1999.

[23] A. A. Kilbas, H. M. Srivastava, and J. J. Trujillo, Theory and Applications of Fractional Differential Equations, Elsevier, San Diego, Calif, USA, 2006.

[24] D. Baleanu, K. Diethelm, E. Scalas, and J. J. Trujillo, Fractional Calculus Models and Numerical Methods, vol. 3 of Series on 
Complexity, Nonlinearity and Chaos, World Scientific, Hackensack, NJ, USA, 2012.

[25] N. T. Shawagfeh, "Analytical approximate solutions for nonlinear fractional differential equations," Applied Mathematics and Computation, vol. 131, no. 2-3, pp. 517-529, 2002.

[26] A. Arikoglu and I. Ozkol, "Solution of fractional differential equations by using differential transform method," Chaos, Solitons and Fractals, vol. 34, no. 5, pp. 1473-1481, 2007.

[27] Y. Li and N. Sun, "Numerical solution of fractional differential equations using the generalized block pulse operational matrix," Computers \& Mathematics with Applications, vol. 62, no. 3, pp. 1046-1054, 2011. 


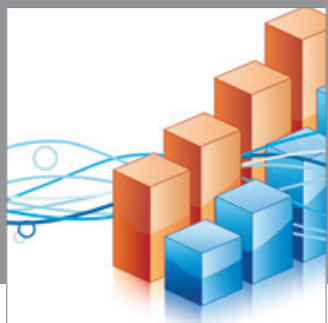

Advances in

Operations Research

mansans

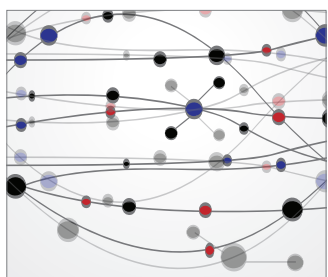

The Scientific World Journal
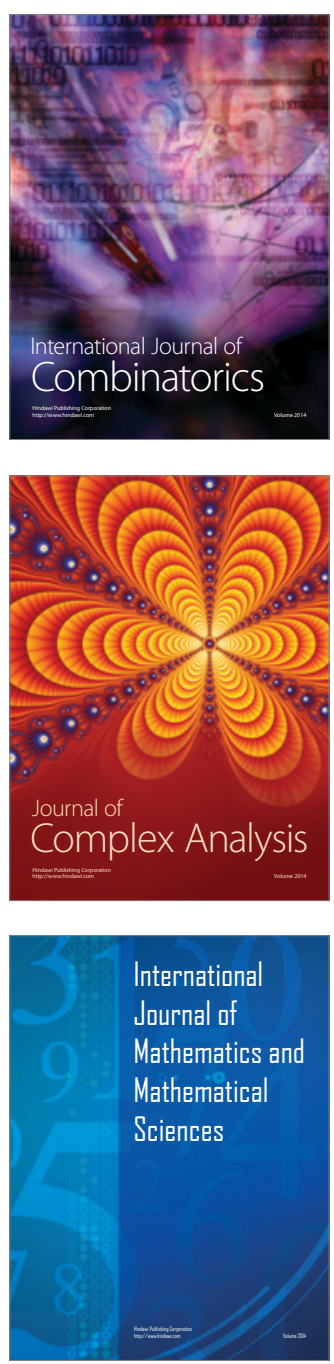
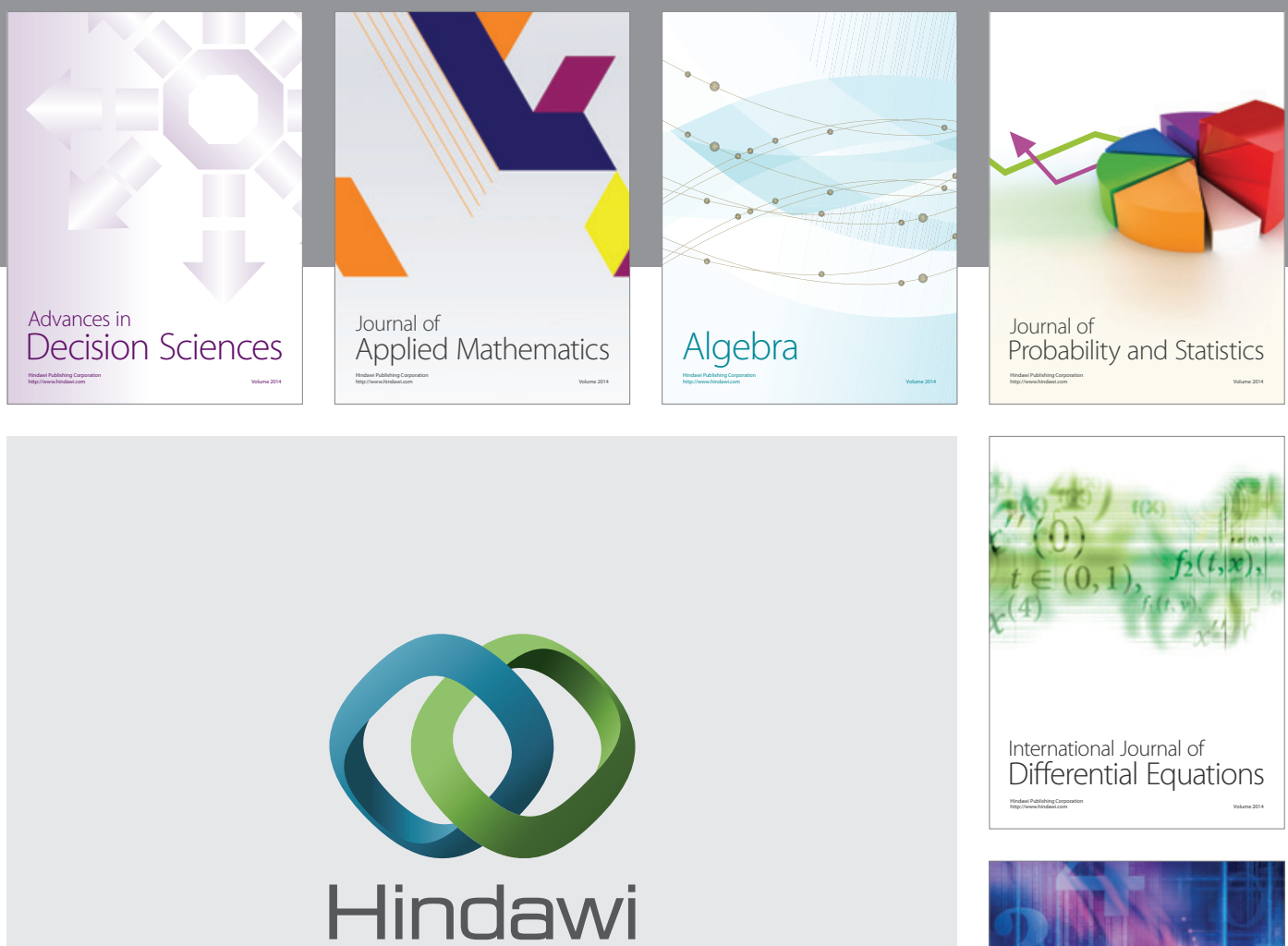

Submit your manuscripts at http://www.hindawi.com
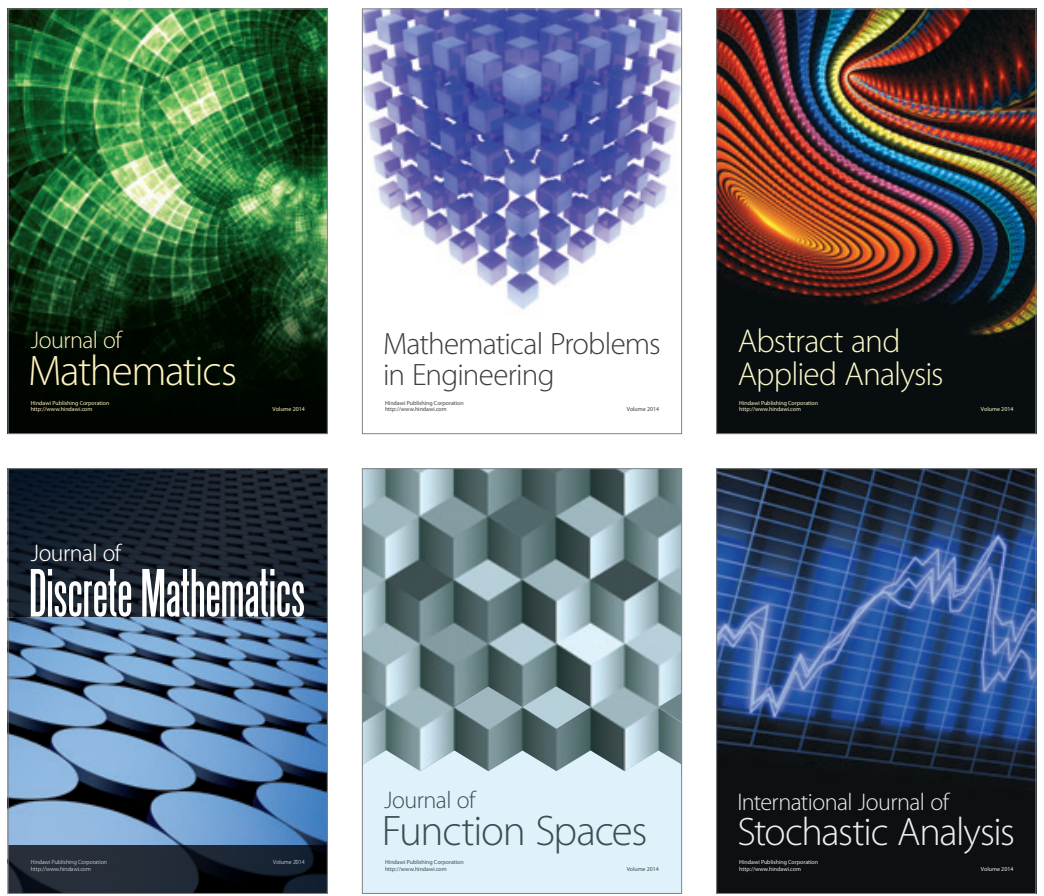

Journal of

Function Spaces

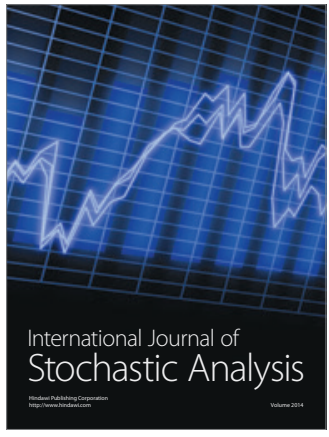

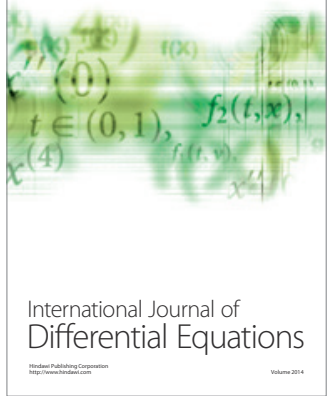
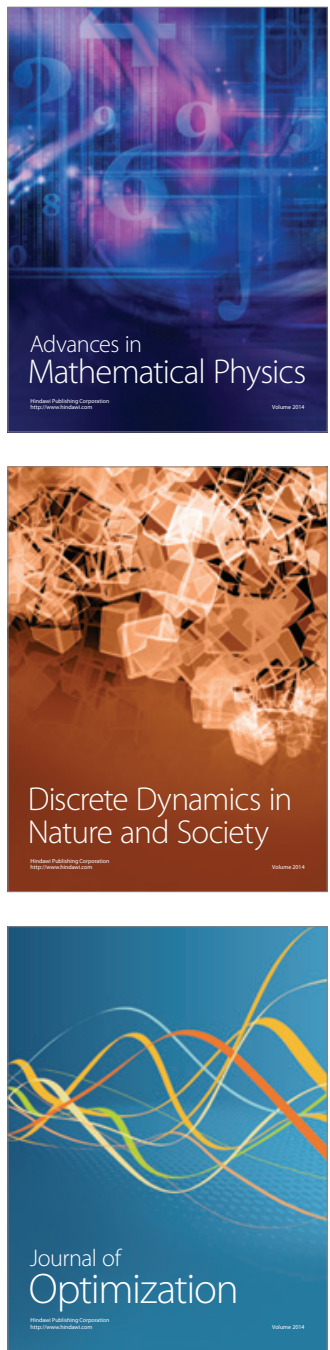